\title{
Incidence, clinical characteristics, and outcomes of nosocomial Enterococcus spp. bloodstream infections in a tertiary-care hospital in Beijing, China: a four-year retrospective study
}

Yangyang Zhang ${ }^{1,2+}$, Mingmei Du ${ }^{3+}$, Yan Chang ${ }^{4}$, Liang-an Chen ${ }^{1 *}$ and Qing Zhang ${ }^{2^{*}}$

\begin{abstract}
Background: Enterococcus spp. are the common cause of nosocomial bloodstream infections (BSIs) with high morbidity and mortality. The purpose of this study was to characterize the incidence, clinical and microbiological features, and mortality of nosocomial enterococcal BSIs at a large Chinese tertiary-care hospital in Beijing, China.

Methods: A retrospective cohort study on adult patients with nosocomial BSIs due to Enterococcus spp. was performed between January 1, 2012, and December 31, 2015 at the Chinese People's Liberation Army General Hospital. Patients' data were gathered by reviewing electronic medical records.

Results: A total of 233 episodes of BSI due to Enterococcus spp. occurred among 224 patients during these 4 years. The overall incidence was 3.9 episodes per 10,000 admissions. Enterococcus faecium (E. faecium) was the major pathogen (74\%, 95\% Cl 68-80\%), followed by Enterococcus faecalis (E. faecalis) (20\%, 95\% Cl 15-25\%). E. faecium showed higher antimicrobial resistance than E. faecalis. The 30-day mortality of nosocomial enterococcal BSI was 24\% (95\% Cl 18-29\%). Predictors for mortality included the Acute Physiology and Chronic Health Evaluation II (APACHE II) score, Charlson comorbidity index (CCI), impaired renal function, prior use of immunosuppressive agents, and appropriate empirical antimicrobial treatment.

Conclusions: This study emphasizes that Enterococcus spp. were major pathogens for nosocomial BSIs and associated with high mortality. Appropriate empirical antimicrobial treatment can improve outcomes. Vancomycin is the best choice for patients with E. faecium BSIs. Penicillins, aminoglycosides, fluoroquinolones, and vancomycin can be considered for patients with $E$. faecalis BSIs.
\end{abstract}

Keywords: Enterococcus, Nosocomial bloodstream infection, Bacteraemia, Epidemiology, Mortality

\footnotetext{
*Correspondence: chenliangan301@163.com; zhangqing_cyfy@163.com

${ }^{\dagger}$ Equal contributors

'Department of Respiratory Medicine, Chinese PLA General Hospital, Fuxing Road No. 28, Beijing 100853, China

${ }^{2}$ Department of Respiratory Medicine, Affiliated Hospital of Chengde Medical University, Nanyingzi Street No. 36, Chengde, Hebei Province 067000, China Full list of author information is available at the end of the article
} 


\section{Background}

BSIs can cause high mortality and result in heavy social and economic burdens. As one of the common grampositive pathogens, Enterococcus spp. are the fourth leading cause of BSIs in North America [1], and are rated as the third most prevalent pathogens of nosocomial BSIs in the United States [2], accounting for $>9.0 \%$ of the BSIs. Several multicentre studies from China and Japan also reported that Enterococcus spp. were the fourth most common BSI pathogens [3-5]. And a systematic review focused on community-acquired BSIs in south and southeast Asia showed that Enterococcus spp. were the third most prevalent gram positive bacteria [6]. The crude mortality rates of enterococcal BSIs ranged between $21.4 \%$ and $64.2 \%$ [7-11]. Risk factors for developing enterococcal BSIs include the presence of comorbidities such as malignancy, diabetes; more severe illness; invasive devices, such as a central intravenous catheter; complicated surgery; solid organ transplantation; and hematopoietic stem cell transplantation [2, 12-16].

Enterococci are innately resistant to cephalosporins, and the commonly used antimicrobial agents such as fluoroquinolones and carbapenems are not recommended. The increasing prevalence of acquired resistance to penicillins and aminoglycosides has been observed in many countries $[17,18]$. Vancomycin-resistant enterococci (VRE) are a great challenge in clinical treatment because there are limited bactericidal options to choose [19].

E. faecalis and E. faecium are the most frequently isolated species, and incidences of both have shown a rising trend, especially for E. faecium $[11,20]$. E. faecium has significantly higher antibiotic resistance rates than E. faecalis and may lead to more serious disease and worse outcome [11, 21, 22].

Although there has been much research on enterococcal BSI, the incidence, species distribution, clinical features, and prognosis vary in different periods and different regions. In addition, nosocomial BSI is different from community-acquired BSI in several aspects, including a poor prognosis [11]. Little data exists regarding nosocomial enterococcal BSIs in China. The purpose of the present study was to characterise the incidence, clinical, and microbiological features, and to identify the predictors of crude mortality in patients with enterococcal BSIs at our hospital. The clinical and microbiological characteristics of E. faecalis and E. faecium were also compared.

\section{Methods}

\section{Study design, hospital setting, and patients}

We performed a retrospective cohort study on adult patients with nosocomial BSIs due to Enterococcus spp. between January 1, 2012, and December 31, 2015, at the
Chinese People's Liberation Army General Hospital (PLAGH), a 2200-bed tertiary-level healthcare hospital in Beijing, China. It is one of the biggest comprehensive hospitals in China, with medical, health care, teaching, and scientific research that serves national military and nonmilitary personnel from across the country.

Eligible patients included all patients aged $\geq 18$ years with at least 1 positive blood culture for Enterococcus spp. In patients with persistent BSIs caused by the same organism, only the first episode was included. If the patients had 2 or more separate BSIs, each infection was considered individually. All patients were identified by searching the real-time nosocomial infection surveillance system (RT-NISS) [23]. This platform utilises data from electronic medical record systems, such as hospital stay, temperature changes, and microbiology results with the application of clinically validated algorithms to identify and classify all of the patients' infections. RT-NISS was developed by the Infection Management and Disease Control Department of PLAGH.

\section{Data collection}

The patients' data were gathered by reviewing electronic medical records. We recorded the demographic data including age and gender. The clinical data collected included: body mass index (BMI), underlying diseases, the CCI score, the APACHE II score in the first $24 \mathrm{~h}$ following the onset of BSIs, the hospitalisation wards, previous exposures (prior hospital stay, previous treatment such as surgical procedures, immunosuppressive agents, chemotherapeutic agents, total parenteral nutrition, mechanical ventilation, renal replacement therapy, antibiotics, or invasive devices within 30 days prior to BSIs), treatment (antibiotic choice), and outcomes (length of hospital stay, and all-cause mortality at 7 days and 30 days). The microbiological data collected included: species of Enterococcus, likely source of BSIs (identified by treating doctors and/or physicians of the Infection Management and Disease Control Department), whether cultures were polymicrobial, and antimicrobial susceptibility results. We collected the annual admission data to calculate incidence rates, which are expressed as the number of BSI episodes per 10,000 hospital admissions.

\section{Definitions}

The diagnosis of enterococcal BSI should meet the following criteria: 1)isolation of Enterococcus spp. from one or more blood cultures; and one of the following 2) fever $\left(>38{ }^{\circ} \mathrm{C}\right)$, chills, or hypotension; and 3 ) eliminated the possibility of contamination during the collection and cultivation of blood samples [24]. Nosocomial BSI was defined as the first positive blood culture obtained $\geq 48 \mathrm{~h}$ after hospital admission and with no evidence of infection at admission $[15,24]$. An episode was defined as 
the positive isolation of enterococcus from at least one blood culture sample from a patient and without the prior blood culture isolating the same bacteria within the previous 30 days $[2,25]$. Onset of BSIs was defined as the date when the blood culture was collected. Polymicrobial BSIs were defined as 2 or more clinically important organisms isolated from 1 single blood culture sample or different blood culture samples within $48 \mathrm{~h}$. Appropriate antimicrobial treatment was defined as an active antimicrobial choice and an adequate dosage within 5 days from the onset. Empirical antimicrobial therapy was defined as the antimicrobial agents given within $24 \mathrm{~h}$ after the onset of BSIs. Active agents were confirmed according to the antimicrobial susceptibility test.

\section{Identification and antibiotic susceptibility testing}

Blood was cultured using BacT/ALERT 3D system (Becton-Dickinson, Sparks, MD, USA) in the microbiology laboratory. Species identification was performed using the VITEK 2 system (BioMérieux, Marcy 1'Étoile, France). Antibiotic susceptibility testing was performed using the VITEK 2 system or the Kirby-Bauer Disk Diffusion method (Oxoid, UK) according to the recommendations proposed by the Clinical and Laboratory Standards Institute (CLSI).

\section{Statistical analysis}

Categorical variables were expressed as frequency counts and percentages with 95\% confidence interval (95\% CI). Continuous variables were expressed as median and interquartile ranges (IQRs). Comparison of categorical variables was performed using Pearson's chi-squared test or Fisher's exact test, and comparison of continuous variables was performed using the Mann-Whitney $U$ test. In order to identify the risk factors associated with mortality, a multivariate logistic regression model with backward method was generated to control the effects of confounding variables. Variables statistically related $(p<0.10)$ to mortality in the univariate logistic analyses were chosen to build the multivariate model. Results with a 2 -tailed $p$-value $<0.05$ were considered to be significant. All statistical analyses were run using SPSS 20.0 software (IBM Corp., Armonk, NY, USA).

\section{Results}

\section{Incidence and species distribution}

In total, 233 episodes of nosocomial BSIs caused by Enterococcus spp. occurred among 224 patients during the 4-year study period. Of the 224 patients who developed enterococcal BSIs, 6 had infections with 2 different species $>30$ days apart and 3 had 2 infections with the same species $>30$ days apart. Seven patients who had infections with 2 different species of enterococcus isolated within $48 \mathrm{~h}$ were excluded because of the interpretative problem.

For the remaining 226 episodes, classification was available in 224 (99\%, 95\% CI 98-100\%). The most common Enterococcus species was E. faecium, which comprised 167 (74\%, 95\% CI 68-80\%) of all episodes. $E$. faecalis was the second largest species, comprising 46 (20\%, 95\% CI 15-25\%) of episodes. Of the remaining episodes, 6 (3\%, 95\% CI 1-5\%) due to E. casseliflavus, $3(1 \%, 95 \%$ CI $0-3 \%)$ due to E. gallinarum, and $2(1 \%$, 95\% CI 0-3\%) due to E. avium. The number of enterococci isolated in 2015 was the highest, and the least was in 2013. The species ratio in each year is shown in Fig. 1.

The overall incidence of nosocomial enterococcal BSIs was 3.9 episodes per 10,000 admissions and the rate fluctuated from 3.3 to 4.4 episodes per 10,000 admissions during the 4 years (3.7 in 2012, 3.3 in 2013, 4.1 in 2014, and 4.4 in 2015). The overall incidence rates of E. faecium and E. faecalis were 2.9 episodes and 0.8 episodes per 10,000 admissions, respectively. Incidence of $E$. faecalis BSI showed a rising trend, while that of $E$. faecium was on the decline, as shown in Fig. 2.

\section{Demographic and clinical characteristics}

Demographics and clinical data were available for all these 226 incident episodes as shown in Table 1. The median age was 65 years (IQR, 54-75), and 142 (63\%, 95\% CI 56-69\%) of the patients were male. The age distribution was right skewed, with the peak incidence in the 50-59 years group (Figure 3). Malignancy was the most common comorbidity (58\%, 95\% CI 51-64\%), followed by cardiovascular disease (50\%, 95\% CI 43-57\%) and pneumonia (33\%, 95\% CI 27-39\%). The median and range of BMI were all within the normal range. The median CCI score was 3 (IQR, 2-6), and $86.7 \%$ of the patients had combined chronic diseases (CCI score $\geq 1$ ). The median APACHE II score was 8 (IQR, 5-18). Some 172 (76\%, 95\% CI 71-82\%) of the episodes occurred in the non-ICU ward, and 54 (24\%, 95\% CI 18-29\%) occurred in the ICU. The median days of hospital stay prior to and

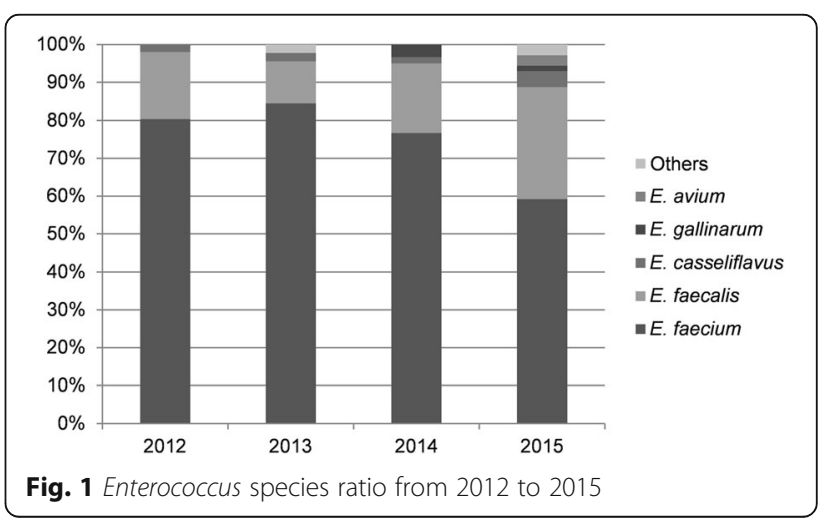




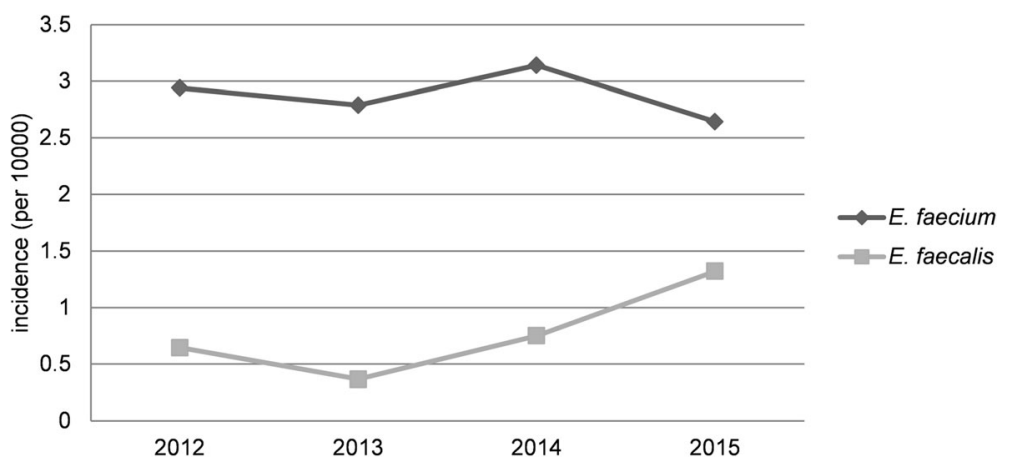

Fig. 2 Incidences of BSIs due to E. faecium and E. faecalis from 2012 to 2015

after the onset of BSI were 15 and 18, respectively. A total of 210 (93\%, 95\% CI 90-96\%) episodes had prior antibiotic exposure and 182 (81\%, 95\% CI 75-86\%) had invasive medical procedures within the 30 days prior to BSI, such as a central intravenous catheter, Indwelling urinary catheter, and drainage tube.

A comparison of nosocomial BSIs according to $E$. faecium and $E$. faecalis in demographics and clinical characteristics is shown in Table 1. Patients with $E$. faecalis BSIs presented a higher BMI, but the median was in the normal range. Patients with E. faecium BSIs showed a higher CCI score than those with $E$. faecalis BSIs (median, 3 vs $2, p=0.014$ ). The median APACHE II score of patients with E. faecium BSIs was higher than in those with $E$. faecalis BSIs, but these were not statistically significant.

\section{Microbiology and antimicrobial therapy}

The source of nosocomial enterococcal BSIs was mainly related to intra-abdominal and central venous catheter, with the number of $92(41 \%, 95 \%$ CI $34-47 \%)$ and 82 (36\%, 95\% CI 30-43\%), respectively. Foci could not be confirmed for 65 (29\%, 95\% CI 23-35\%) episodes. A total of 21 (9\%, 95\% CI 5-13\%) episodes had polymicrobial infections.

The microbiological data of BSIs due to E. faecium and E. faecalis are presented in Table 2. There was no difference in the sources of BSI between these 2 species. Patients with $E$. faecium BSIs were more likely to have polymicrobial infections than those with E. faecalis ( $12 \%$ vs $2 \%, p=0.048)$. No vancomycin-resistant $E$. faecalis isolate was found, and 7 (4\%, 95\% CI 1-7\%) vancomycinresistant $E$. faecium isolates were identified. E. faecium isolates had higher resistance rates than $E$. faecalis to ampicillin ( $86 \%$ vs $9 \%, p<0.001)$, erythromycin $(78 \%$ vs $56 \%, p=0.009)$, and ciprofloxacin ( $86 \%$ vs $39 \%$, $p<0.001)$.

Before obtaining the report of antibiotic susceptibility test reports, 98 (43\%, 95\% CI 37-50\%) patients were treated with effective antibiotics. Patients with E. faecalis BSIs were more likely to get effective treatment by the empirical use of antibiotics ( $57 \%$ vs $37 \%, p=0.018$ ).

\section{Outcomes}

The median length of hospital stay was 34 days (IQR, 23-55). There was no statistical difference in the length of stay between patients with E. faecium BSIs (median, 35) and those with E. faecalis BSIs (median, 34). The total 7-day and 30-day mortality rates were $11 \%$ (95\% CI 7-15\%) and 24\% (95\% CI 18-29\%), respectively. The 7-day and 30-day mortality rates for E. faecium BSIs were higher than E. faecalis BSIs (13\% vs $9 \%, 25 \%$ vs $17 \%)$, but both had no statistical significance. Patients with malnutrition showed a higher risk of 7-day and 30-day mortality than patients with normal BMI.

Univariate analyses of predictors for 7-day mortality and 30-day mortality are shown separately in Table 3 . The 7-day mortality was associated with pneumonia, impaired renal function, CCI, APACHE II score, ICU residence, prior exposure to immunosuppressive agents and central intravenous catheter, and appropriate empirical antimicrobial treatment. The predictors for 30-day mortality were similar to those for 7-day mortality, except for surgery and renal replacement therapy prior to the onset of BSI within 30 days.

In the multivariate logistic regression model (Table 4), the risk factors for 7-day mortality included increasing APACHE II scores (OR 1.2, 95\% CI 1.1-1.3), and the use of immunosuppressive agents prior to the onset of enterococcal BSIs (OR 4.6, 95\% CI 1.1-19.2); the risk factors for 30-day mortality included impaired renal function (OR 3.3, 95\% CI 1.1-9.8), high CCI (OR 1.3, 95\% CI 1.1-1.6) and APACHE II scores (OR 1.3, 95\% CI 1.2-1.4), and prior exposure to immunosuppressive agents (OR 7.3, 95\% CI 1.8-29.0). Appropriate empirical antimicrobial therapy was a protective factor for both 7-day (OR 0.2, 95\% CI 0.1-0.7) and 30-day mortality (OR 0.2, 95\% CI 0.1-0.4). 
Table 1 Demographic and clinical characteristics of patients with enterococcal BSIs

\begin{tabular}{|c|c|c|c|c|}
\hline & $\begin{array}{l}\text { Total } \\
(n=226)\end{array}$ & $\begin{array}{l}\text { E. faecium } \\
(n=167)\end{array}$ & $\begin{array}{l}\text { E. faecalis } \\
(n=46)\end{array}$ & $p$-value \\
\hline \multicolumn{5}{|l|}{ Demographics } \\
\hline Age & $65(54-75)$ & $65(55-75)$ & $66(52-79)$ & 0.450 \\
\hline Male gender & $142(63,56-69)$ & $104(62,55-70)$ & $31(67,53-81)$ & 0.524 \\
\hline BMI, median & $23(20-26)$ & $22(20-25)$ & $24(23-26)$ & 0.006 \\
\hline \multicolumn{5}{|l|}{ Comorbidities } \\
\hline Malignancy & $130(58,51-64)$ & $95(57,49-64)$ & $26(57,42-71)$ & 0.965 \\
\hline Cardiovascular disease & $113(50,43-57)$ & $78(47,39-54)$ & $28(61,46-76)$ & 0.089 \\
\hline Pneumonia & $74(33,27-39)$ & $60(36,29-43)$ & $14(30,17-44)$ & 0.488 \\
\hline Diabetes mellitus & $68(30,24-36)$ & $45(27,20-34)$ & $18(39,24-54)$ & 0.109 \\
\hline Impaired liver function & $52(23,17-29)$ & $41(25,18-31)$ & $7(15,4-26)$ & 0.180 \\
\hline Cerebrovascular disease & $41(18,13-23)$ & $34(20,14-27)$ & $5(11,2-20)$ & 0.141 \\
\hline Impaired renal function & $30(13,9-18)$ & $22(13,8-18)$ & $7(15,4-26)$ & 0.720 \\
\hline Hemiplegia & $27(12,8-16)$ & $24(14,9-20)$ & $2(4,0-10)$ & 0.066 \\
\hline Neutropenia & $14(6,3-9)$ & $12(7,3-11)$ & $2(4,0-10)$ & 0.492 \\
\hline $\mathrm{CCl}$, median & $3(2-6)$ & $3(2-6)$ & $2(1-4)$ & 0.014 \\
\hline APACHE II score, median & $8(5-11)$ & $8(5-11)$ & $7(5-12)$ & 0.501 \\
\hline \multicolumn{5}{|l|}{ Hospitalization ward } \\
\hline Medical & $88(39,33-45)$ & $68(41,33-48)$ & $14(30,17-44)$ & 0.204 \\
\hline Surgical & $84(37,31-44)$ & $56(34,26-41)$ & $22(48,33-63)$ & 0.075 \\
\hline ICU & $54(24,18-29)$ & $43(26,19-32)$ & $10(22,9-34)$ & 0.578 \\
\hline \multicolumn{5}{|l|}{ Length of hospital stay, median days } \\
\hline Prior hospital stay & $15(9-27)$ & $16(10-28)$ & $14(7-25)$ & 0.065 \\
\hline Hospital stay after onset of BSIs & $18(8-29)$ & $18(8-29)$ & $18(8-33)$ & 0.906 \\
\hline \multicolumn{5}{|l|}{ Previous treatment } \\
\hline Antibiotic exposure & $210(93,90-96)$ & $160(96,93-99)$ & $41(89,80-98)$ & 0.082 \\
\hline Total parenteral nutrition & $84(37,31-44)$ & $60(36,29-43)$ & $22(48,33-63)$ & 0.142 \\
\hline Mechanical ventilation & $87(38,32-45)$ & $63(38,30-45)$ & $20(43,29-58)$ & 0.479 \\
\hline Surgery & $74(33,27-39)$ & $49(29,22-36)$ & $20(43,29-58)$ & 0.070 \\
\hline Chemotherapeutic agent & $29(13,8-17)$ & $24(14,9-20)$ & $3(7,0-14)$ & 0.153 \\
\hline Immunosuppressive agent & $18(8,4-12)$ & $16(10,5-14)$ & $2(4,2-10)$ & 0.259 \\
\hline Renal replacement therapy & $15(7,3-10)$ & $12(7,3-11)$ & $3(7,0-14)$ & 0.876 \\
\hline \multicolumn{5}{|l|}{ Invasive devices } \\
\hline Central intravenous catheter & $102(45,39-52)$ & $82(49,41-57)$ & $16(35,20-49)$ & 0.084 \\
\hline Indwelling urinary catheter & $104(46,39-53)$ & $75(45,37-53)$ & $25(54,39-69)$ & 0.256 \\
\hline Endotracheal intubation & $86(38,32-44)$ & $60(36,29-43)$ & $22(48,33-63)$ & 0.142 \\
\hline Peripheral intravenous catheter & $69(31,24-37)$ & $50(30,23-37)$ & $14(30,17-44)$ & 0.948 \\
\hline Tracheostomy tube & $20(9,5-13)$ & $15(9,5-13)$ & $4(9,0-17)$ & 0.952 \\
\hline
\end{tabular}

Data are presented as $\mathrm{n}(\%, 95 \% \mathrm{Cl})$ or median (IQR)

Significant variables are appeared in bold and italics text

\section{Discussion}

This study focused on the incidence and characteristics of nosocomial enterococcal BSI in one of the biggest comprehensive hospitals in China. Two multicentre studies in China reporting on the species distribution and antibiotic resistance of clinical isolates from blood cultures showed that Enterococcus spp. were the fourth most common pathogens [3, 4]. But, these 2 studies did not involve the species distribution, clinical information, and prognosis. A single-centre study published recently, 


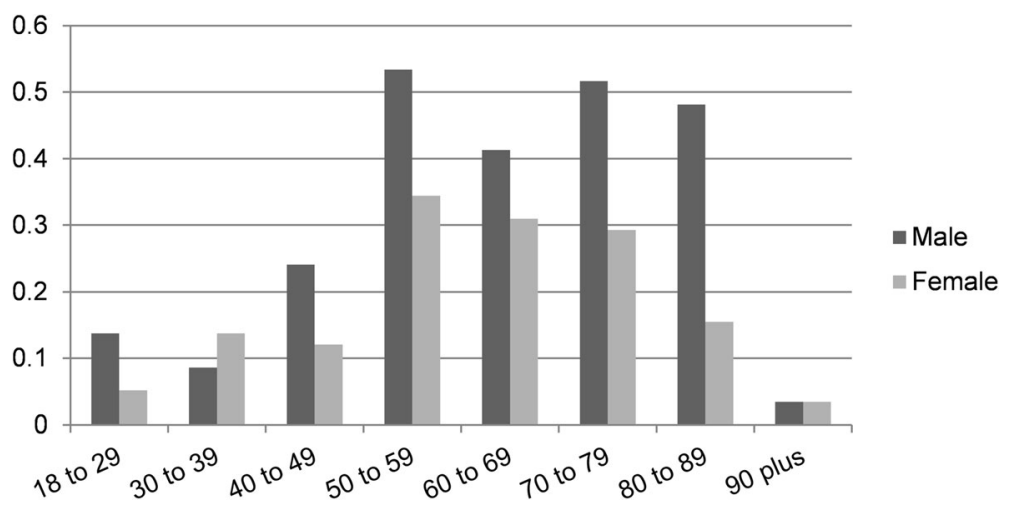

Fig. 3 Age and gender distribution of patients with enterococcal BSIs

also devoted to explore the features of Enterococcus spp. BSI in a teaching hospital of China [26]. But the sample size (64 episodes) was smaller than our study, and all nosocomial, health care associated, and community acquired enterococcal BSIs were included.

In our 4-year study, the incidence rate of nosocomial enterococcal BSIs fluctuated from 3.3 to 4.4 episodes per 10,000 admissions. This is similar to an earlier study conducted from 1995 to 2002 at 42 US hospitals [2], but is higher than 2 recent reports from Denmark (1.96/ $10,000)$ and Canada $(0.69 / 10,000)[11,15]$. But these were both population-based studies. To our knowledge, there are no such reference data about the incidence of nosocomial enterococcal BSIs in China.

In accordance with other studies, patients with enterococcal BSIs were commonly associated with complications, such as malignant, cardiovascular diseases, diabetes mellitus, or chronic kidney disease [11, 15, 22, 27]. We

Table 2 Comparison of the microbiological characteristics and treatment of patients with E. faecium and E. faecalis BSIs

\begin{tabular}{|c|c|c|c|}
\hline & E. faecium $(n=167)$ & E. faecalis $(n=46)$ & $p$-value \\
\hline \multicolumn{4}{|l|}{ Source of BSIs } \\
\hline Intra-abdominal & $65(39,31-46)$ & $19(41,27-56)$ & 0.770 \\
\hline Unknown & $53(32,25-39)$ & $10(22,9-34)$ & 0.188 \\
\hline Central venous catheter & $30(18,12-24)$ & $10(22,9-34)$ & 0.562 \\
\hline Genitourinary & $11(7,3-10)$ & $3(7,0-14)$ & 0.987 \\
\hline Pneumonia & $4(2,0-5)$ & $3(7,0-14)$ & 0.165 \\
\hline Others & $4(2,0-5)$ & $1(2,0-7)$ & 0.930 \\
\hline \multicolumn{4}{|l|}{ Type of BSIs } \\
\hline Polymicrobial & $20(12,7-17)$ & $1(2,0-7)$ & 0.048 \\
\hline \multicolumn{4}{|l|}{ Antibiotic resistance ${ }^{a}$} \\
\hline Ampicillin (137 vs 46) ${ }^{b}$ & $118(86,80-92)$ & $4(9,0-7)$ & $<0.001$ \\
\hline Gentamicin (93 vs 21) ${ }^{b}$ & $49(53,42-63)$ & $7(33,11-55)$ & 0.109 \\
\hline Tetracycline (105 vs 36) ${ }^{b}$ & $50(48,38-57)$ & $22(61,44-78)$ & 0.162 \\
\hline Erythromycin (105 vs 36) ${ }^{\mathrm{b}}$ & $82(78,70-86)$ & $20(56,39-73)$ & 0.009 \\
\hline Ciprofloxacin (137 vs 46) b & $118(86,80-92)$ & $18(39,24-54)$ & $<0.001$ \\
\hline Vancomycin (167 vs 46$)^{b}$ & $7(4,1-7)$ & $0(0,0)$ & 0.158 \\
\hline \multicolumn{4}{|l|}{ Treatment after the onset of BSIs } \\
\hline Appropriate antimicrobial treatment & $157(94,90-98)$ & $43(93,86-100)$ & 0.893 \\
\hline Appropriate empirical treatment & $62(37,30-45)$ & $26(57,42-71)$ & 0.018 \\
\hline
\end{tabular}

Data are presented as $\mathrm{n}(\%, 95 \% \mathrm{Cl})$ or median (IQR)

Significant variables are appeared in bold and italics text

${ }^{a}$ Not all agents listed tested in all isolates

${ }^{b}$ The numbers in parentheses represent the total numbers of $E$. faecium and E. faecalis isolates performed susceptibility test 


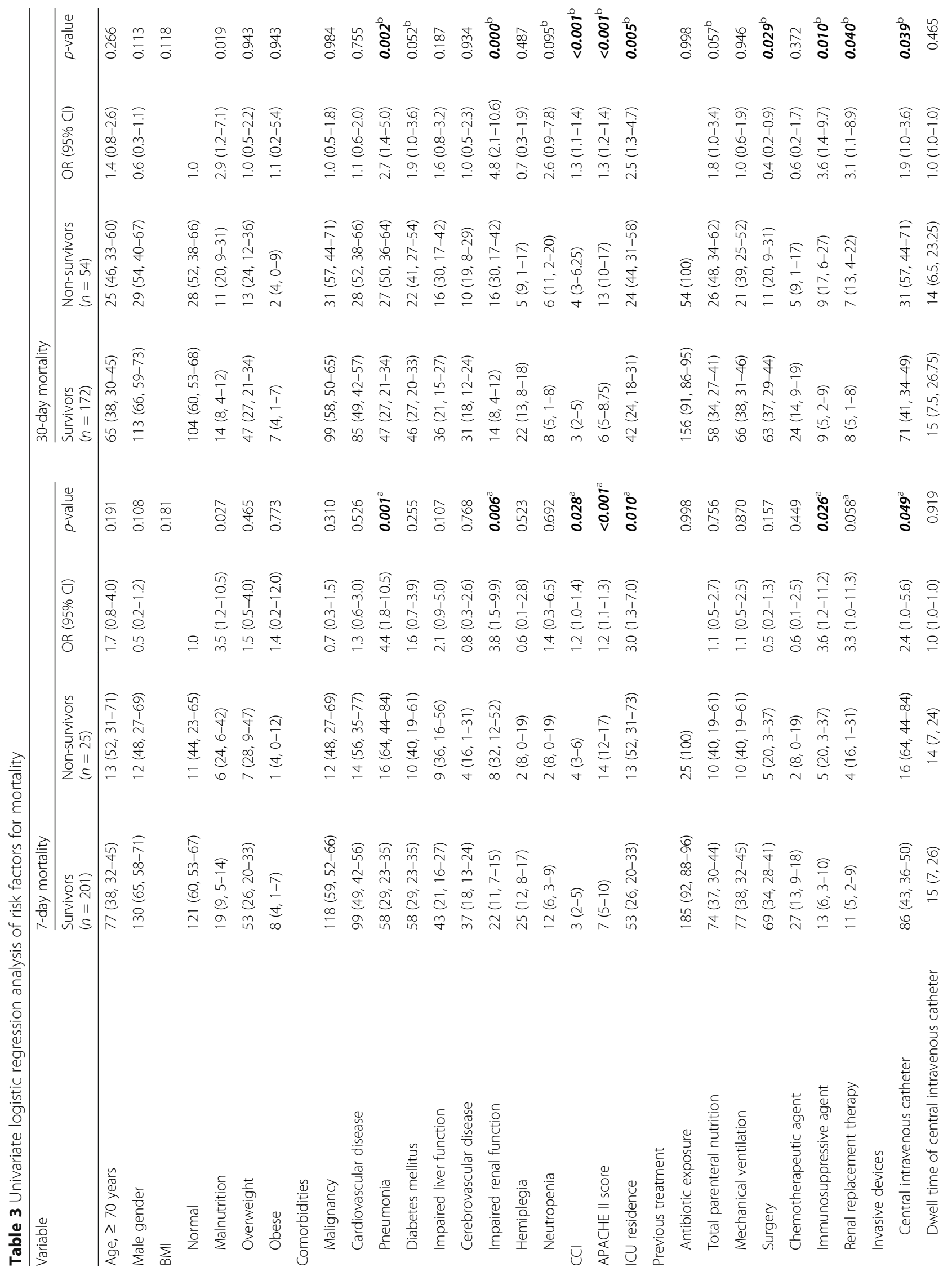




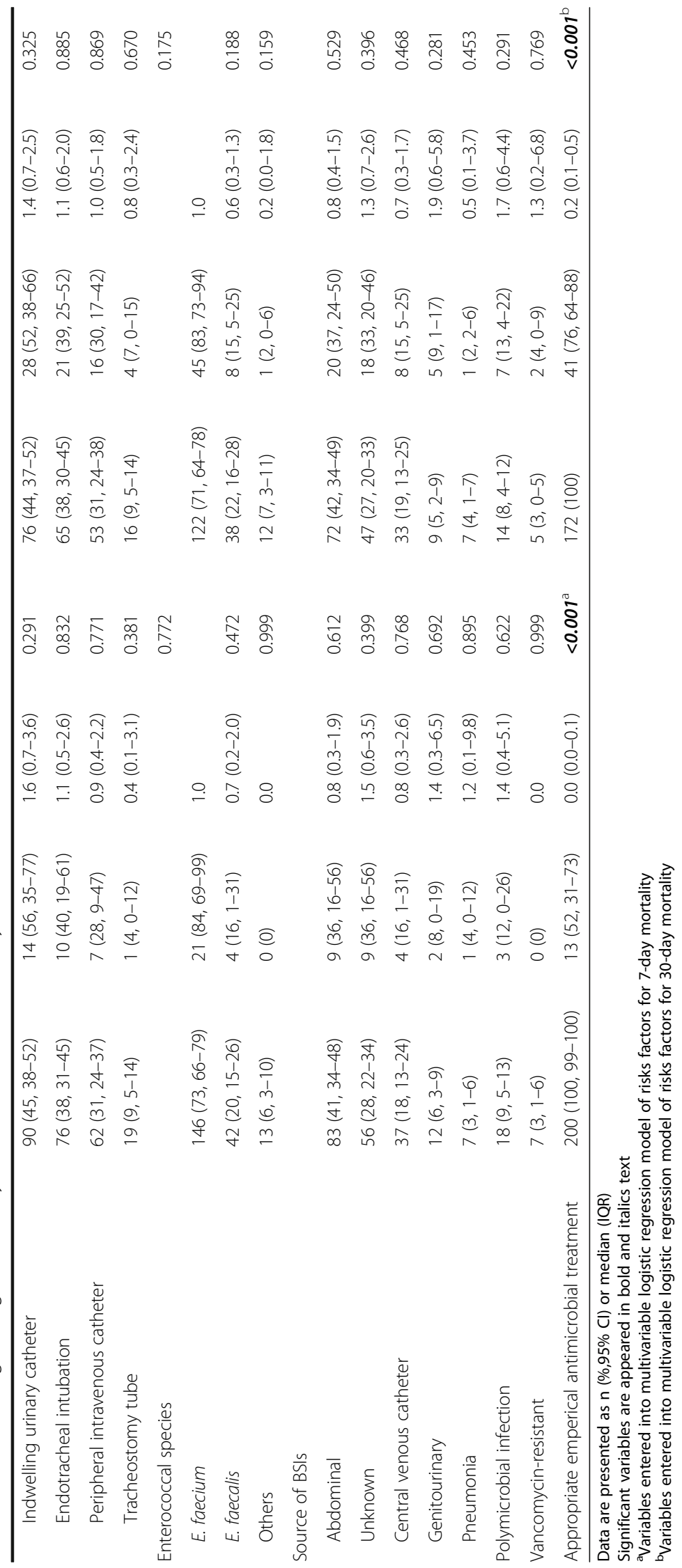


Table 4 Multivariate logistic regression models of risk factors for mortality

\begin{tabular}{|c|c|c|c|c|}
\hline \multirow[t]{2}{*}{ Variable } & \multicolumn{2}{|c|}{ 7-day mortality } & \multicolumn{2}{|c|}{ 30-day mortality } \\
\hline & OR $(95 \% \mathrm{Cl})$ & $p$-value & OR $(95 \% \mathrm{Cl})$ & $p$-value \\
\hline Diabetes mellitus & - & - & $2.1(0.9-5.4)$ & 0.103 \\
\hline Impaired renal function & $2.1(0.6-6.9)$ & 0.220 & $3.3(1.1-9.8)$ & $0.030^{\mathrm{b}}$ \\
\hline $\mathrm{CCl}$ & $1.2(0.9-1.5)$ & 0.080 & $1.3(1.1-1.6)$ & $0.003^{b}$ \\
\hline APACHE II score & $1.2(1.1-1.3)$ & $<0.001^{a}$ & $1.3(1.2-1.4)$ & $<0.001^{b}$ \\
\hline Prior use of immunosuppressive agents & $4.6(1.1-19.2)$ & $0.036^{\mathrm{a}}$ & $7.3(1.8-29.0)$ & $0.005^{\mathrm{b}}$ \\
\hline Appropriate empirical antimicrobial therapy & $0.2(0.0-0.7)$ & $0.010^{\mathrm{a}}$ & $0.2(0.1-0.4)$ & $<0.001^{b}$ \\
\hline
\end{tabular}

Significant variables are appeared in bold and italics text

${ }^{a}$ Variables entered the final multivariable logistic regression model of risks factors for 7-day mortality

${ }^{b}$ Variables entered the final multivariable logistic regression model of risks factors for 30-day mortality

found that pneumonia was also a major comorbidity of nosocomial enterococcal BSIs at our hospital.

E. faecalis was reported to be the most common pathogen for nosocomial enterococcal BSIs in most investigations, and the ratio of E. faecium was no more than $50 \%[1,11,20,28]$. However, a constant increase in E. faecium BSI rates was observed [11, 29], and the incidence of E. faecium BSIs exceeded E. faecalis BSI in 2009 in a 14-year study conducted at a Swiss tertiary hospital [29]. At our hospital, the incidence of nosocomial E. faecium BSIs was significantly higher than that of E. faecalis BSIs $(2.9 / 10,000$ vs $0.8 / 10,000)$. The total vancomycin resistance rate of all isolates was $3 \%$, and $4 \%$ for E. faecium in our study. All of the vancomycinresistant isolates were $E$. faecium. This vancomycin resistance rate is higher than those in the Danish study (1.9\%) [11], but lower than many studies in other countries. The SCOPE project carried out between 1995 and 1996 at 49 US hospitals reported the VRE rate was between $9.5 \%$ to $20.6 \%$ in nosocomial enterococcal BSIs from different hospitals [30]. The 1997 SENTRY program reported that $14.1 \%$ of enterococcal BSIs were VRE in the United States [1]. A prospective nationwide surveillance study at Brazilian hospitals, which published in 2011, showed that the VRE rate was as high as 25\% [9]. Therefore, the species distribution and antimicrobial resistance varies geographically.

Abdominal infections, central venous catheters, and unknown sources were the most common foci of enterococcal BSIs in the present study, is similar to the prior studies [28]. However, no infective endocarditis was observed in our study. There may be 2 reasons to explain this difference. First, the study population was different, as we were focused on nosocomial infection. Another reason was that echocardiography was not a routine examination for patients with BSIs in our hospital, so this could lead to missed diagnosis.

Our data showed that patients with nosocomial E. faecalis BSIs were more likely to get appropriate empirical treatment than those with E. faecium BSIs. This may be explained by the low resistance rate of $E$. faecalis to many antimicrobial agents, such as ampicillin (9\%), gentamicin (33\%), and ciprofloxacin (39\%).

In the present study, the 30-day mortality of nosocomial enterococcal BSIs was $24 \%$, and the early mortality (7-day) accounted for half. This is lower than many prior reports $[5,8,9,12,31]$, but similar to a study focusing on vancomycin-susceptible enterococcal BSIs [22]. This may be due to selection bias of the study population, or may be a reflection of medical progress. Mortality was higher in patients with higher CCI and APACHE II scores, prior use of immunosuppressive agents, and complicated with impaired renal function. In other reports, the risk factors for mortality of patients with enterococcal BSIs may also include advanced age, pulmonary infection, malignancy, species, polymicrobial infection, ampicillin resistance, high-level gentamicin resistance, and vancomycin resistance [11, 15, 22]. Although controversial, we confirm that the severity of underlying diseases is the most important factor, especially for early morbidity.

The present study has several limitations that should be taken into consideration. First, our study was a retrospective study, the collection of clinical data depended on medical records rather than interviews and clinical examinations at the onset of infection by unified training doctors of our research group. Second, as a single-centre study, it could lead to an inevitable selection bias, and the multivariate logistic analysis might be affected by the small sample size. Third, not all isolates did all the same antimicrobial agent sensitivity test, so we could not incorporate all the antimicrobial agents resistance in predicting mortality risk factors in order to avoid selection bias.

\section{Conclusions}

Enterococci were major pathogens for nosocomial BSIs and were associated with high mortality,especially for 
patients combined with chronic diseases. Increased CCI and APACHE II scores, prior use of immunosuppressive agent and complicated with impaired renal function were risk factors for morality. E. faecium were more common than E. faecalis at our hospital. For patients with E. faecium BSIs who conditions were complicated with serious underlying diseases, vancomycin is the best choice; for patients with E. faecalis BSIs, penicillins, aminoglycosides, and fluoroquinolones could also be considered besides vancomycin.

\section{Abbreviations \\ APACHE II: Acute Physiology and Chronic Health Evaluation II; BMI: Body mass index; BSIs: Bloodstream infections; CCl: Charlson comorbidity index; $\mathrm{Cl}$ : Confidence interval; IQRs: Interquartile ranges; OR: Odds ratio; PLAGH: Chinese People's Liberation Army General Hospital; RT-NISS: Real-time nosocomial infection surveillance system; VRE: Vancomycin-resistant enterococci}

\section{Acknowledgments}

We thank all the colleagues in the Infection Management and Disease Control Department for making this study possible.

\section{Funding}

This study was funded by grants from the Key Projects in the Military Program during the Twelfth Five-year Plan Period (BWS1 1 J057). The grant agency didn't involve in the design of the study, collection, analysis and interpretation of data, and writing or revising the manuscript.

\section{Availability of data and materials}

The datasets generated during the current study are not publicly available due to avoid disclosure the individual privacy of the patients, but are available from the corresponding author on reasonable request.

\section{Authors' contributions}

YZ and MD conceived this study, collected clinical data, interpreted the results, wrote and revised the manuscript. YC participated in collecting data and data statistics. LC and QZ participated in the study design and revised the manuscript. All authors read and approved the final manuscript.

\section{Ethics approval and consent to participate}

This study was approved by the local institutional review board. For this type of study formal consent is not required.

\section{Consent for publication}

Not applicable.

\section{Competing interests}

None of authors declare conflicts of interest relevant to this article.

\section{Publisher's Note}

Springer Nature remains neutral with regard to jurisdictional claims in published maps and institutional affiliations.

\footnotetext{
Author details

'Department of Respiratory Medicine, Chinese PLA General Hospital, Fuxing Road No. 28, Beijing 100853, China. ${ }^{2}$ Department of Respiratory Medicine, Affiliated Hospital of Chengde Medical University, Nanyingzi Street No. 36, Chengde, Hebei Province 067000, China. ${ }^{3}$ Department of Infection Management and Disease Control, Chinese PLA General Hospital, Beijing 100853, China. ${ }^{4}$ Department of Respiratory Medicine, The General Hospital of the PLA Rocket Force, Xinjiekou Street No. 16, Beijing 100088, China.
}

Received: 28 March 2017 Accepted: 27 June 2017

Published online: 04 July 2017

\section{References}

1. Pfaller MA, Jones RN, Doern GV, Sader HS, Kugler KC, Beach ML. Survey of blood stream infections attributable to gram-positive cocci: frequency of occurrence and antimicrobial susceptibility of isolates collected in 1997 in the United States, Canada, and Latin America from the SENTRY antimicrobial surveillance program. SENTRY participants group. Diagn Microbiol Infect Dis. 1999;33(4):283-97.

2. Wisplinghoff $H$, Bischoff $T$, Tallent $S M$, Seifert $H$, Wenzel RP, Edmond MB. Nosocomial bloodstream infections in US hospitals: analysis of 24,179 cases from a prospective nationwide surveillance study. Clin Infect Dis. 2004;39(3): 309-17.

3. Huang YC, Xie Y, Chen ZX, Liu B, Fei Y, Du Y, Shan B, Kang M. Bloodstream infections in southwestern China: 2012 Whire union report on bacterial susceptibility to antibiotics. Sichuan da xue xue bao Yi xue ban. 2015;46(1): $75-81$.

4. Guanghui ZD LI, Fu WANG, Zhidong HU, Quan LI, Ziyong SUN. The distribution and antibiotic resistance of clinical isolates from blood culture in 2012 CHINET surveillance program in China. Chin J Chemother. 2014; 14(6):474-81.

5. Nagao M. A multicentre analysis of epidemiology of the nosocomial bloodstream infections in Japanese university hospitals. Clin Microbio Infect. 2013;19(9):852-8.

6. Deen J, von Seidlein L, Andersen F, Elle N, White NJ, Lubell Y. Communityacquired bacterial bloodstream infections in developing countries in south and southeast Asia: a systematic review. Lancet Infect Dis. 2012;12(6):480-7.

7. DiazGranados CA, Zimmer SM, Klein M, Jernigan JA. Comparison of mortality associated with vancomycin-resistant and vancomycin-susceptible enterococcal bloodstream infections: a meta-analysis. Clin Infect Dis. 2005; 41(3):327-33.

8. Caballero-Granado FJ, Becerril B, Cuberos L, Bernabeu M, Cisneros JM, Pachon J. Attributable mortality rate and duration of hospital stay associated with enterococcal bacteremia. Clin Infect Dis. 2001:32(4):587-94.

9. Marra AR, Camargo LF, Pignatari AC, Sukiennik T, Behar PR, Medeiros EA Ribeiro J, Girao E, Correa L, Guerra C, Brites C, Pereira CA, Carneiro I, Reis M, de Souza MA, Tranchesi R, Barata CU, Edmond MB. Nosocomial bloodstream infections in Brazilian hospitals: analysis of 2,563 cases from a prospective nationwide surveillance study. J Clin Microbiol. 2011;49(5):1866-71.

10. Linden PK, Pasculle AW, Manez R, Kramer DJ, Fung JJ, Pinna AD, Kusne S. Differences in outcomes for patients with bacteremia due to vancomycinresistant Enterococcus faecium or vancomycin-susceptible E. Faecium. Clin Infect Dis. 1996;22(4):663-70

11. Pinholt M, Ostergaard C, Arpi M, Bruun NE, Schonheyder HC, Gradel KO, Sogaard M, Knudsen JD. Incidence, clinical characteristics and 30-day mortality of enterococcal bacteraemia in Denmark 2006-2009: a populationbased cohort study. Clin Microbiol Infect. 2014;20(2):145-51.

12. Noskin GA, Peterson LR, Warren JR (1995) Enterococcus faecium and Enterococcus faecalis bacteremia: acquisition and outcome. Clin Infect Dis 1995;20(2):296-301

13. Peel T, Cheng AC, Spelman T, Huysmans M, Spelman D. Differing risk factors for vancomycin-resistant and vancomycin-sensitive enterococcal bacteraemia. Clin Microbiol Infect. 2012;18(4):388-94.

14. Tavadze M, Rybicki L, Mossad S, Avery R, Yurch M, Pohlman B, Duong H, Dean R, Hill B, Andresen S, Hanna R, Majhail N, Copelan E, Bolwell B, Kalaycio M, Sobecks R. Risk factors for vancomycin-resistant enterococcus bacteremia and its influence on survival after allogeneic hematopoietic cell transplantation. Bone Marrow Transplant. 2014;49(10):1310-6.

15. Billington EO, Phang SH, Gregson DB, Pitout JD, Ross T, Church DL, Laupland KB, Parkins MD. Incidence, risk factors, and outcomes for Enterococcus spp. blood stream infections: a population-based study. Int J Infect Dis. 2014:26:76-82

16. Berenger BM, Doucette K, Smith SW. Epidemiology and risk factors for nosocomial bloodstream infections in solid organ transplants over a 10-year period. Transpl Infect Dis. 2016;18(2):183-90.

17. Bush LM, Calmon J, Cherney CL, Wendeler M, Pitsakis P, Poupard J, Levison ME, Johnson CC. High-level penicillin resistance among isolates of enterococci. Implications for treatment of enterococcal infections. Ann Intern Med. 1989;110(7):515-20. 
18. Patterson JE, Zervos MJ. High-level gentamicin resistance in Enterococcus: microbiology, genetic basis, and epidemiology. Rev Infect Dis. 1990;12(4): 644-52.

19. Barber KE, King ST, Stover KR, Pogue JM. Therapeutic options for vancomycin-resistant enterococcal bacteremia. Expert Rev Anti-Infect Ther. 2015;13(3):363-77.

20. Bhavnani SM, Drake JA, Forrest A, Deinhart JA, Jones RN, Biedenbach DJ, Ballow $\mathrm{CH}$. A nationwide, multicenter, case-control study comparing risk factors, treatment, and outcome for vancomycin-resistant and -susceptible enterococcal bacteremia. Diagn Microbiol Infect Dis. 2000;36(3):145-58.

21. Conde-Estevez D, Grau S, Albanell J, Terradas R, Salvado M, Knobel H. Clinical characteristics and outcomes of patients with vancomycinsusceptible Enterococcus faecalis and Enterococcus faecium bacteraemia in cancer patients. Eur J Clin Microbiol Infect Dis. 2011;30(1):103-8.

22. McBride SJ, Upton A, Roberts SA. Clinical characteristics and outcomes of patients with vancomycin-susceptible Enterococcus faecalis and Enterococcus faecium bacteraemia-a five-year retrospective review. Eur J Clin Microbiol Infect Dis. 2010;29(1):107-14.

23. Du M, Xing Y, Suo J, Liu B, Jia N, Huo R, Chen C, Liu Y. Real-time automatic hospital-wide surveillance of nosocomial infections and outbreaks in a large Chinese tertiary hospital. BMC Med Inform Decis Mak. 2014;14:9.

24. Garner JS, Jarvis WR, Emori TG, Horan TC, Hughes JM. CDC definitions for nosocomial infections. Am J Infect Control. 1988;16:128-40.

25. Satlin MJ, Soave R, Racanelli AC, Shore TB, van Besien K, Jenkins SG, Walsh $\mathrm{TJ}$. The emergence of vancomycin-resistant enterococcal bacteremia in hematopoietic stem cell transplant recipients. Leuk Lymphoma. 2014;55(12): 2858-65.

26. Z Zheng JX, Li H, Pu ZY, Wang HY, Deng XB, Liu XJ, Deng QW, Yu ZJ. Bloodstream infections caused by Enterococcus spp: A10-year retrospective analysis at a tertiary hospital in China. J Huazhong Univ Sci Technolog Med Sci. 2017;37(2):257-63.

27. Vigani AG, Oliveira AM, Bratfich OJ, Stucchi RS, Moretti ML. Clinical, epidemiological, and microbiological characteristics of bacteremia caused by high-level gentamicin-resistant Enterococcus faecalis. Braz I Med Biol Res. 2008:41(10):890-5.

28. Hamada Y, Magarifuchi H, Oho M, Kusaba K, Nagasawa Z, Fukuoka M, Yamakuchi H, Urakami T, Aoki Y. Clinical features of enterococcal bacteremia due to ampicillin-susceptible and ampicillin-resistant enterococci: an eightyear retrospective comparison study. J Infect Chemother. 2015;21 (7):527-30

29. Weisser M, Capaul S, Dangel M, Elzi L, Kuenzli E, Frei R, Widmer A. Additive effect of Enterococcus faecium on Enterococcal bloodstream infections: a 14-year study in a Swiss tertiary hospital. Infect Control Hosp Epidemiol. 2013;34(10):1109-12.

30. Jones RN, Marshall SA, Pfaller MA, Wilke WW, Hollis RJ, Erwin ME, Edmond MB, Wenzel RP. Nosocomial enterococcal blood stream infections in the SCOPE program: antimicrobial resistance, species occurrence, molecular testing results, and laboratory testing accuracy. SCOPE hospital study group. Diagn Microbiol Infect Dis. 1997;29(2):95-102.

31. Landry SL, Kaiser DL, Wenzel RP. Hospital stay and mortality attributed to nosocomial enterococcal bacteremia: a controlled study. Am J Infect Control. 1989;17(6):323-9.

\section{Submit your next manuscript to BioMed Central and we will help you at every step:}

- We accept pre-submission inquiries

- Our selector tool helps you to find the most relevant journal

- We provide round the clock customer support

- Convenient online submission

- Thorough peer review

- Inclusion in PubMed and all major indexing services

- Maximum visibility for your research

Submit your manuscript at www.biomedcentral.com/submit

) Biomed Central 